\title{
Gaps in the Numbers of Vertices of Cubical Polytopes, I
}

\author{
G. Blind ${ }^{1}$ and R. Blind ${ }^{2}$ \\ ${ }^{1}$ Mathematisches Institut B, Universität Stuttgart, \\ Pfaffenwaldring 57, D-70550 Stuttgart (Vaihingen), Germany \\ ${ }^{2}$ Waldburgstrasse 88, D-70563 Stuttgart (Vaihingen), Germany
}

\begin{abstract}
A cubical polytope is a convex polytope of which very facet is a combinatorial cube. We ask for the numbers which occur as vertex numbers of $d$-dimensional cubical polytopes, and we show, as a first step, that every cubical $d$-polytope for even $d \geq 4$ has an even number of vertices.
\end{abstract}

\section{Introduction}

Let $P$ be a convex $d$-polytope in $\mathbb{R}^{d}$ and let $f_{j}(P)$ be the number of $j$-dimensional faces of $P(0 \leq j \leq d-1)$. A basic problem is to determine the possible $f_{j}(P)$ for all convex polytopes $P$ or for interesting classes of convex polytopes $P$. For example, any 3-polytope $P$ with $f_{1}(P)=7$ does not exist $[5$, p. 187$]$.

In the case of simple polytopes $P$, McMullen's conditions (see, e.g., p. 131 of [2]) give a complete characterization of the $f$-vector $\left(f_{0}(P), \ldots, f_{d-1}(P)\right)$ of $P$. Using these conditions Perles (lecture in Oberwolfach, 1990) studied the possible numbers of vertices of simple polytopes. It is easily seen that in odd dimensions only even numbers of vertices occur, and he asked for the smallest number past which all (resp. all even) numbers are realizable as vertex numbers of simple polytopes.

Analogously, we ask here for the possible numbers of vertices of cubical polytopes, that is, convex polytopes of which every facet is a combinatorial cube. It is known [1] that a cubical polytope has at least $2^{d}$ vertices whenever $d>2$. Jockusch [6] conjectured that there will be some gaps for numbers slightly larger than $2^{d}$ but that all numbers of vertices will be possible past a certain point. In contrast to this we show:

Theorem. Let $P$ be a cubical d-polytope in $\mathbb{R}^{d}$ for $d \geq 4$, and let $d$ be even. Then the number $f_{0}(P)$ of vertices of $P$ is even. 
In dimension $d=3$ cubical polytopes with odd numbers of vertices exist, namely, with $2^{d}=8,10,11,12, \ldots$ vertices. We do not know whether cubical polytopes with an odd number of vertices in odd dimensions $d \geq 5$ exist.

We regard combinatorially isomorphic polytopes as identical. For example, an $n$-cube here means any combinatorial $n$-cube.

We need the following properties of the cube and some related terminology. Let $F$ be any facet of an $n$-cube $C_{n}$, then one and only one facet $F^{\prime}$ disjoint from $F$ exists. $F^{\prime}$ is said to be the the facet opposite to $F$ in $C_{n}$. Let $K$ be a $j$-face of $C_{n}(0 \leq j \leq n-1)$. Let $F_{1}, \ldots, F_{n-j}$ be the facets containing $K$, and let $F_{1}^{\prime}, \ldots, F_{n-j}^{\prime}$ be the corresponding opposite facets. Then $K^{\prime}:=F_{1}^{\prime} \cap \cdots \cap F_{n-j}^{\prime}$ is also a $j$-face and is called the j-face opposite to $K$ in $C_{n}$. Let $F$ and $F^{\prime}$ be opposite facets of $C_{n}$. Every edge not contained in $F$ or $F^{\prime}$ intersects $F$ in a vertex $v$ and $F^{\prime}$ in a vertex $v^{\prime}$. Vertices $v$ and $v^{\prime}$ are called related vertices of the facets $F$ and $F^{\prime}$.

Following the usual notation, vert $P$ is the set of vertices of $P$. For a set $S \subset P$, a vertex of $S$ means a vertex of $P$ lying in $S$. Thus vert $S:=\operatorname{vert} P \cap S$. If $M$ is a finite set, $|M|$ is the number of its elements. A subfacet (of a facet $F$ ) of $P$ is a $(d-2$ )-face of $P$ (contained in $F$ ).

The proof of the theorem uses shellings and colorings of cubical polytopes.

\section{Shellings of Cubical Polytopes}

Let $P$ be a convex polytope and let $\partial P$ be the boundary complex of $P$. A shelling of $\partial P$ is a labeling $\left\{F_{1}, \ldots, F_{m}\right\}$ of the $m=f_{d-1}(P)$ facets of $P$ so that, for $s=2, \ldots, m-1$, the set $B_{s}:=F_{s} \cap \bigcup_{i=1}^{s=1} F_{i}$ is homeomorphic to a $(d-2)$-ball. This implies that $\bigcup_{i=1}^{s} F_{i}$ is homeomorphic to a $(d-1)$-ball for $s=1, \ldots, m-1$. It is well known that $\partial P$ is shellable (see, e.g., [3] or p. 173 of [7]).

For $s=1, \ldots, m$, define $M_{s}:=\bigcup_{i=1}^{s} F_{i}$, and put $M_{0}:=\varnothing$. A vertex $v$ of $M_{s}$ is called an inner vertex of $M_{s}$ if all the facets of $P$ containing $v$ lie in $M_{s}$, and is called an outer vertex of $M_{s}$ otherwise. We have $M_{s}=F_{s} \cup M_{s-1}$, and this union is the sth step of the shelling.

We are interested in vert $M_{s} \backslash$ vert $M_{s-1}$, the set of vertices added in the sth step. These are outer vertices of $M_{s}$. We are also interested in how the sth step changes the set of outer vertices; thus besides vert $M_{s} \backslash$ vert $M_{s-1}$ we are interested in the set of outer vertices of $M_{s-1}$ becoming inner vertices of $M_{s}$.

For $s=m$ we have vert $P=\operatorname{vert} M_{m}=$ vert $M_{m-1}$, so

$$
\text { vert } M_{m} \backslash \text { vert } M_{m-1}=\varnothing \text {, }
$$

and the outer vertices of $M_{m-1}$ are just the vertices of $F_{m}$. For $s=1$ we have

$$
\text { vert } M_{1}=\text { vert } F_{1} \text {, }
$$

and all the vertices of $M_{1}=F_{1}$ are outer vertices of $M_{1}$.

In order to examine the sth step of the shelling for $s=2, \ldots, m-1$, consider $F_{s}$ and $B_{s}=F_{s} \cap M_{s-1}$. Clearly, vert $M_{s} \backslash$ vert $M_{s-1}$ consists of the vertices of $F_{s}$ 
not lying in $B_{s}$. Furthermore, the vertices of $M_{s-1}$ becoming inner vertices of $M_{s}$ lie in $B_{s}$. Since $M_{s-1}$ is homeomorphic to a $(d-1)$-ball, a vertex $v$ of $B_{s}$ becomes an inner vertex of $M_{s}$ if and only if all the subfacets of $F_{s}$ containing $v$ are contained in $B_{s}$.

Now let $P$ be a cubical polytope. Then we can describe $B_{s}$ more precisely: Let $K$ be a $k$-face of $F_{s}(0 \leq k \leq d-2)$. Then the cube $F_{s}$ has the following subfacets:

- the subfacets containing $K$,

-the subfacets disjoint from $K$, that is, the subfacets opposite to subfacets containing $K$, and

--if $k>0$, the subfacets intersecting but not containing $K$; they intersect $K$ in $(k-1)$-faces.

$B_{s}$ is homeomorphic to a $(d-2)$-ball, so $B_{s}$ is the union of subfacets of $F_{s}$. Thus, induction on the number of subfacets of $F_{s}$ contained in $B_{s}$ shows: if $B_{s}$ does not contain pairs of opposite subfacets of $F_{s}$, then $B_{s}$ is the union of all the subfacets of $F_{s}$ containing a certain $k$-face of $F_{s}(0 \leq k \leq d-2)$.

Hence, according to the type of $B_{s}$, we have to distinguish three possibilities:

Type 1: $B_{s}$ contains a pair $S_{1}$ and $S_{2}$ of opposite subfacets of $F_{s}$. Then all the vertices of $F_{s}$ lie in $B_{s}$, so that

$$
\text { vert } M_{s} \backslash \text { vert } M_{s-1}=\varnothing
$$

We claim that the set of outer vertices of $M_{s-1}$ becoming inner vertices of $M_{s}$ consists of pairs of related vertices of $S_{1}$ and $S_{2}$. To see this let $v_{1} \in S_{1}$ be an inner vertex of $M_{s}$. Then, by the above, all the subfacets of $F_{s}$ passing through $v_{1}$ are contained in $B_{s}$. Let $v_{2}$ be the vertex of $S_{2}$ related to $v_{1} \in S_{1}$. The subfacets of $F_{s}$ containing $v_{2}$ are $S_{2}$ and some subfacets containing $v_{1}$, and so they are all contained in $B_{s}$. Hence, by the above, $v_{2}$ is also an inner vertex of $M_{s}$, which completes the proof.

Type 2: $B_{s}$ is the union of the subfacets of $F_{s}$ containing a certain $k$-face $K$ of $F_{s}$, with $1 \leq k \leq d-2$. Let $K^{\prime}$ be the $k$-face of $F_{s}$ opposite to $K$ in $F_{s}$. Then the vertices of $F_{s}$ not lying in $B_{s}$ are exactly the vertices of $K^{\prime}$, so that

$$
\operatorname{vert} M_{s} \backslash \text { vert } M_{s-1}=\operatorname{vert} K^{\prime}
$$

We claim that no outer vertices of $M_{s-1}$ become inner vertices of $M_{s}$. To see this let $v$ be a vertex of $B_{s}$ or, more generally, of $F_{s}$. Since $k \geq 1$, a subfacet $S_{v}$ of $F_{s}$ containing $v$ and not containing $K$ exists. Hence $S_{v} \not \subset B_{s}$. Thus, by the above, $v$ is not an inner vertex of $M_{s}$, which completes the proof.

Type 3: $B_{s}$ is the union of the subfacets of $F_{s}$ passing through a certain vertex $v_{0}$ of $F_{s}$. Let $v_{0}^{\prime}$ be the vertex of $F_{s}$ opposite to $v_{0}$ in $F_{s}$. Then $v_{0}^{\prime}$ is the only vertex of $F_{s}$ not lying in $B_{s}$, so that

$$
\text { vert } M_{s} \backslash \text { vert } M_{s-1}=\left\{v_{0}^{\prime}\right\} \text {. }
$$


We claim that the set of outer vertices of $M_{s-1}$ becoming inner vertices of $M_{s}$ is $\left\{v_{0}\right\}$. In fact, $v_{0}$ is an outer vertex of $M_{s-1}$, and since all the subfacets of $F_{s}$ passing through $v_{0}$ are contained in $B_{s}, v_{0}$ is an inner vertex of $M_{s}$. Conversely, let $v \neq v_{0}$ be a vertex of $B_{s}$. Then $v$ is contained in a subfacet through $v_{0}^{\prime}$ and so is an outer vertex of $M_{s}$, which completes the proof.

\section{Colorings of the Set of Vertices of a Polytope}

If $P$ is a polytope, vert $P$ is said to be well-colored if the vertices of $P$ are colored with two colors so that every two adjacent vertices have different colors.

It is easy to see that vert $P$ is well-colorable if and only if the length of each circuit on $P$ is even (a circuit on $P$ is a sequence $\left\{x_{0}, x_{1}, \ldots, x_{k}=x_{0}\right\}$ of successively adjacent vertices of $P$, and $k$ is its length).

Lemma. Let $P$ be a d-polytope, $d \geq 2$, so that every 2-face of $P$ has an even number of edges. Then vert $P$ is well-colorable.

This lemma is known (see p. 154 of [4], but the proof given there has a gap, since a circuit on $P$ need not be homeomorphic to the boundary of a circle). However, the lemma follows from the shellability of $\partial P$ :

Proof. We show that each circuit on $P$ has even length. This is true for $d=2$ and we prove it generally by induction on $d$. We assume, therefore, that it is true for $d-1 \geq 2$. Let $P$ be a $d$-polytope so that every 2 -face of $P$ has an even number of edges. Let $\left\{F_{1}, \ldots, F_{m}\right\}\left(m=f_{d-1}(P)\right)$ be a shelling of $\partial P$. Then, by the induction hypothesis, every circuit on $F_{s}$ has even length $(s=1, \ldots, m)$. Define $M_{s}:=\bigcup_{i=1}^{s} F_{i}$ for $s=1, \ldots, m$. Since vert $P=$ vert $M_{m-1}$ and since all the edges of $P$ lie in $M_{m-1}$, the assertion is true if all the circuits on $M_{s}$ have even length for $s=1, \ldots, m-1$. Because $M_{1}=F_{1}$ this is true for $s=1$, and we prove it generally by induction on $s$.

We assume, therefore, that every circuit on $M_{s-1}$ has even length for $1 \leq s-1 \leq m-2$, and we recall that every circuit on $F_{s}$ has even length. Now let $C=\left\{x_{0}, \ldots, x_{k}=x_{0}\right\}$ be a circuit on $M_{s}=F_{s} \cup M_{s-1}$ with points in both $F_{s}$ and $M_{s-1}$. Then $C$ intersects $B_{s}:=F_{s} \cap M_{s-1}$ in some successive points $x_{i_{0}}, \ldots$, $x_{i_{j}}=x_{i_{0}}$. Since $B_{s}$ is homeomorphic to a $(d-2)$-ball, $B_{s}$ is the union of subfacets of $F_{s}$ and is connected. Thus a circuit $C_{0}$ on $B_{s}$ passing $x_{i_{0}}, \ldots, x_{i_{j}}=x_{i_{0}}$ successively exists. Then the two circuits $C$ and $C_{0}$ split into two circuits lying entirely in $F_{s}$ (resp. $M_{s-1}$ ). Since these two circuits and $C_{0}$ have even length, so has $C$, which completes the proof.

\section{Proof of the Theorem}

Let $P$ be a cubical $d$-polytope, with $d \geq 4$. The number of vertices of $P$ is

$$
\begin{aligned}
\mid \text { vert } P|=| \text { vert } M_{m} \mid= & \left(\mid \text { vert } M_{m}|-| \text { vert } M_{m-1} \mid\right)+\cdots+\left(\mid \text { vert } M_{s}|-| \text { vert } M_{s-1} \mid\right) \\
& +\cdots+\left(\mid \text { vert } M_{2}|-| \text { vert } M_{1} \mid\right)+\mid \text { vert } M_{1} \mid .
\end{aligned}
$$


According to Section 2 we have $\mid$ vert $M_{m}|-|$ vert $M_{m-1} \mid=0$ and $\mid$ vert $M_{1} \mid=$ $2^{d-1}$. Furthermore, $\mid$ vert $M_{s}|-|$ vert $M_{s-1} \mid$ is odd if and only if the $s$ th step of the shelling is of type $3(2 \leq s \leq m-1)$. Hence, $\mid$ vert $P \mid$ is even if and only if the number of steps of type 3 is even.

In order to study this, let vert $P$ be well-colored with the two colors black and white. Let $B\left(M_{s}\right)$ be the number of black outer vertices of $M_{s}$, let $W\left(M_{s}\right)$ be the number of white outer vertices of $M_{s}$, and put $B W\left(M_{s}\right):=B\left(M_{s}\right)-W\left(M_{s}\right)$, $s=1, \ldots, m$.

Clearly,

$$
\begin{aligned}
B W\left(M_{m-1}\right)= & \left(B W\left(M_{m-1}\right)-B W\left(M_{m-2}\right)\right)+\cdots+\left(B W\left(M_{s}\right)-B W\left(M_{s-1}\right)\right) \\
& +\cdots+\left(B W\left(M_{2}\right)-B W\left(M_{1}\right)\right)+B W\left(M_{1}\right) .
\end{aligned}
$$

According to Section 2, the outer vertices of $M_{m-1}$ are just the vertices of $F_{m}$, so that $B W\left(M_{m-1}\right)=0$. All the vertices of $M_{1}=F_{1}$ are outer vertices, so that $B W\left(M_{1}\right)=0$. Thus

$$
\begin{aligned}
\left(B W\left(M_{m-1}\right)-B W\left(M_{m-2}\right)\right)+\cdots+\left(B W\left(M_{s}\right)-B W\left(M_{s-1}\right)\right) & \\
& +\cdots+\left(B W\left(M_{2}\right)-B W\left(M_{1}\right)\right)=0 .
\end{aligned}
$$

According to Section 2, the sth step in the shelling $(2 \leq s \leq m-1)$ changes the set of outer vertices as follows. It lets vert $M_{s} \backslash$ vert $M_{s-1}$ appear as new outer vertices, and it lets the outer vertices of $M_{s-1}$ becoming inner vertices of $M_{s}$ disappear. Thus, if the sth step is of type 1 or $2, B W\left(M_{s}\right)-B W\left(M_{s-1}\right)=0$. However, if the sth step is of type 3, precisely one new outer vertex $v_{0}^{\prime}$ appears, and precisely one outer vertex $v_{0}$ disappears, and $v_{0}$ and $v_{0}^{\prime}$ are opposite vertices of $F_{s}$. Thus if the dimension $d$ of $P$ is even, that is, if the dimension $(d-1)$ of $F_{s}$ is odd, then $v_{0}$ and $v_{0}^{\prime}$ have different colors. Thus $B W\left(M_{s}\right)-B W\left(M_{s-1}\right)= \pm 2$ if the sth step in the shelling is of type 3 , and if dimension $d$ is even.

Together with $(*)$ this implies that the number of steps of type 3 is even whenever $d$ is even, which completes the proof of the theorem.

\section{Remarks}

Pursuing the total number of black vertices and the total number of white vertices of $M_{s}(s=1, \ldots, m)$ in the shelling we obtain:

Corollary 1. Let $P$ be a cubical d-polytope in $\mathbb{R}^{d}$ for $d \geq 4$, and let $d$ be even. If the vertices of $P$ are well-colored with the two colors black and white, then $P$ has as many white vertices as black ones.

The $f$-vector $\left(f_{0}(P), \ldots, f_{d-1}(P)\right)$ of a cubical polytope satisfies a certain set of linear equations, see, e.g., p. 156 of [5]. It is easy to deduce from these 
equations that, for even-dimensional cubical polytopes, $f_{0}(P)-f_{1}(P)$ is even. So we have:

Corollary 2. Let $P$ be a cubical d-polytope in $\mathbb{R}^{d}$ for $d \geq 4$, and let $d$ be even. Then the number $f_{1}(P)$ of edges of $P$ is even.

\section{References}

1. G. Blind and R. Blind, Convex polytopes without triangular faces, Israel J. Math. 71 (1990), 129-134.

2. A. Brøndsted, An Introduction to Convex Polytopes, Springer-Verlag, New York, 1983.

3. H. Bruggesser and P. Mani, Shellable decompositions of cells and spheres, Math. Scand. 29 (1972), 197-205.

4. H. S. M. Coxeter, Regular Polytopes, Dover Publications, New York, 1973.

5. B. Grünbaum, Convex Polytopes, Interscience, London, 1967.

6. W. Jockusch, The lower and upper bound problems for cubical polytopes, Discrete Comput. Geom. 9 (1993), 159-163.

7. P. McMullen and G. C. Shephard, Convex Polytopes and the Upper Bound Conjecture, Cambridge University Press, Cambridge, 1971.

Received July 24, 1992, and in revised form May 19, 1993. 